\title{
ARM Single Column Model (SCM) Intercomparison Procedures for Case 1: Summer 1995 SCM IOP
}

\author{
R.T. Cederwall \\ S.K. Krueger
}

April 1998

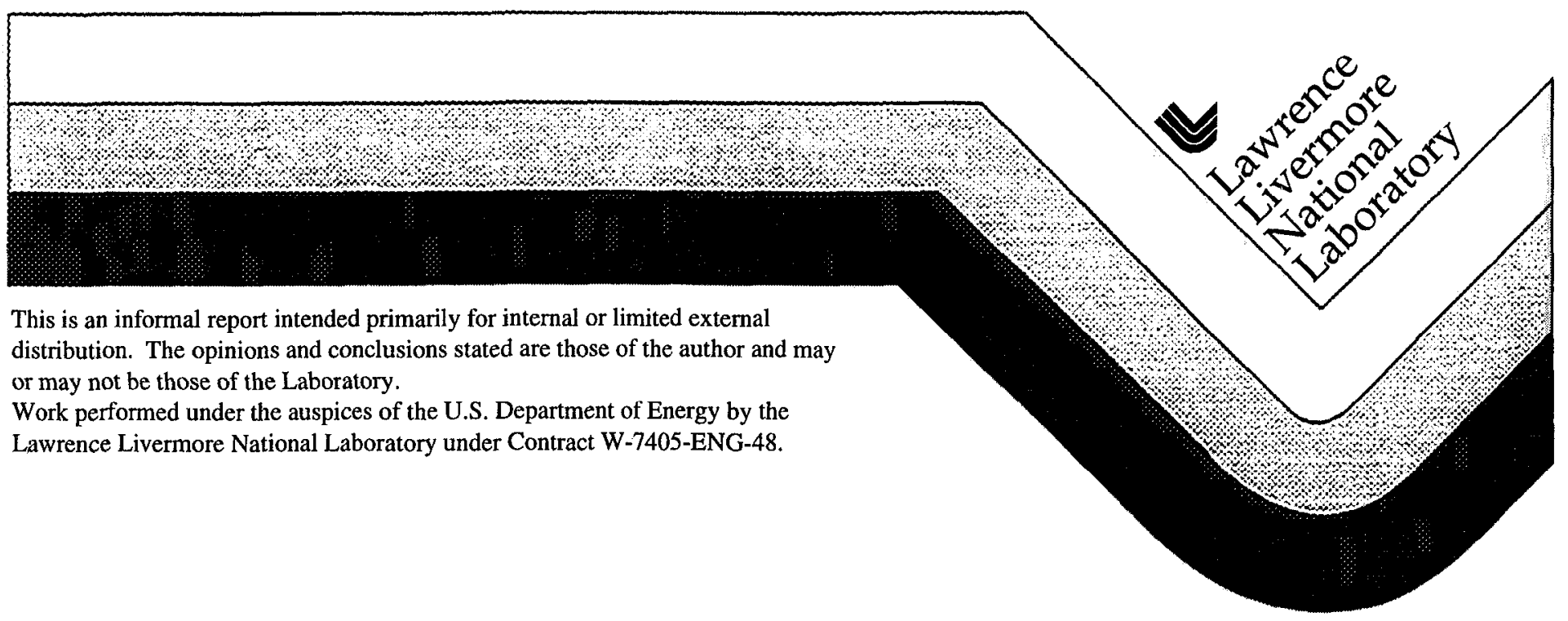


This document was prepared as an account of work sponsored by an agency of the United States Government. Neither the United States Government nor the University of California nor any of their employees, makes any warranty, express or implied, or assumes any legal liability or responsibility for the accuracy, completeness, or usefuiness of any information, apparatus, product, or process disclosed, or represents that its use would not infringe privately owned rights. Reference herein to any specific commercial product, process, or service by trade name, trademark, manufacturer, or otherwise, does not necessarily constitute or imply its endorsement, recommendation, or favoring by the United States Government or the University of California. The views and opinions of authors expressed herein do not necessarily state or reflect those of the United States Government or the University of California, and shall not be used for advertising or product endorsement purposes.

This report has been reproduced directly from the best available copy.

Available to DOE and DOE contractors from the Office of Scientific and Techuical Information P O. Box 62, Oak Ridge, TN 37831

Prices available from (423) 576-8401

Available to the public from the National Technical Information Service

US Department of Commerce 5285 Port Royal Rd.,

Springfield, VA 22161 


\title{
ARM Single Column Model (SCM) Intercomparison Procedures for Case 1: Summer 1995 SCM IOP
}

\author{
Richard T. Cederwall ${ }^{1}$ and Steven K. Krueger ${ }^{2}$
}

A PostScript version of this file is available via anonymous ftp from dev.ec.arm.gov in directory outgoing/scm_intercomp/case1_9507; you can get one of three files:

$$
\begin{array}{lrr}
\text { scm-intercomp-case1-doc.ps } & \text { (uncompressed -- } 1006 \text { KBytes), } \\
\text { scm-intercomp-case1-doc.ps.z } & \text { (compressed -- } & 552 \text { KBytes), } \\
\text { scm-intercomp-case1-doc.ps.gz } & \text { (gzipped -- } & 521 \text { KBytes). }
\end{array}
$$

The large file size is due to the need to include fonts to assist you in printing out these files. See FTP procedures in section 2.4.1 below for further instructions. Information about the SCM Intercomparison is also on the World Wide Web (http://wetfly.llnl.gov/scm/scm_intercomp). You can find the above documentation, as well as access to data files and associated plots.

\section{Introduction}

\subsection{ARM Objectives}

The Atmospheric Radiation Measurement (ARM) Program is a multi-laboratory, interagency program that was created in 1989 with funding from the U.S. Department of Energy (DOE). The ARM Program is part of DOE's effort to resolve scientific uncertainties about global climate change with a specific focus on improving the performance of general circulation models (GCMs) used for climate research and prediction. These improved models will help scientists better understand the influences of human activities on the earth's climate.

The objectives of the ARM Program are:

(1) to relate observed atmospheric radiation to the temperature and composition of the atmosphere, especially water vapor and clouds, across a wide range of climatologically relevant possibilities, and

(2) to develop and test improved parameterizations of radiation and radiative interactions with water vapor, aerosols, and clouds, for use in GCMs.

\footnotetext{
${ }^{1}$ Lawrence Livermore National Laboratory

${ }^{2}$ University of Utah
} 


\subsection{SCM Working Group Objectives}

The Single-Column Model (SCM) Working Group is focused on the second objective of ARM. SCMs provide an economical means for testing parameterizations of physical processes active within a column of cells in a GCM. The SCM Working Group seeks to identify the data requirements for the ARM SCM research, and to facilitate scientific advances in this area by promoting collaborations among ARM Science Team members through common activities and workshops. The SCM Intercomparison is the most recent SCM research activity of the SCM Working Group.

\subsection{SCM Intercomparison}

The SCM methodologies developed in the ARM Program have matured to the point where an intercomparison will help identify the strengths and weaknesses of the various approaches. In the past, SCM Working Group members have shared results at SCM workshops, but the various runs have used different data sets, and different supporting data so that definitive comparisons cannot be made among them.

\subsubsection{Objectives}

In this intercomparison study, we seek to provide a common set of forcing data, and supporting data, for running the SCMs. In this way, the differences in results will be due to differences in the SCM methodologies, not the input data sets. We seek to evaluate the results from the participating models to address several issues, including the adequacy of the methods for deriving estimates of forcing terms from ARM data, the effects of differents ways of prescribing the large-scale forcing, and the performance of the SCMs themselves.

\subsubsection{Description}

The intercomparison will be based on observations collected during the SCM IOP that took place from 17 July to 4 August 1995 at the SGP ARM CART. The comparison runs will cover 18 July (00Z) to 3 August (23Z). This IOP was chosen for the initial case study since it was convectively driven, and some SCM Working Group members had experience with simulating the period. Unfortunately the objective analyses were degraded by the incomplete sounding data set from the southern boundary facility (B6 - Purcell). Data sets from B6 were more complete in later SCM IOPs, so we expect more representative analyses when case studies are selected from them. Future SCM IOP case studies will be chosen that characterize effects of strong synoptic forcing, as well as convective forcing. 


\section{Numerical Experiment Protocol}

\subsection{General Description for All Models}

The descriptions below of initial conditions, upper and lower boundary conditions, and large-scale forcing apply to all models, 1D single-column models (SCMs) as well as 2D and 3D cloud-resolving models (CRMs). Aspects that apply only to CRMs will follow these descriptions.

\subsubsection{Initial conditions}

The initial profiles are based on the observed SGP CART areal averages of temperature, water vapor mixing ratio, and horizontal wind velocity. These are provided as functions of pressure at DP mb intervals from BOT to TOP mb. For values derived by the LLNL objective analysis, $\mathrm{DP}=10 \mathrm{mb}, \mathrm{BOT}=960 \mathrm{mb}$, and TOT $=100 \mathrm{mb}$. For the values derived by Zhang's variational analysis, $\mathrm{DP}=50 \mathrm{mb}, \mathrm{BOT}=965 \mathrm{mb}$, and TOT $=115$ $\mathrm{mb}$. The values at BOT $\mathrm{mb}$ represent those of the layer that starts at the surface. The values provided must be interpolated to each model's vertical grid levels.

To obtain values for model levels below BOT mb by interpolation, assume that $\bar{\theta}, \bar{q}$, $\bar{u}$, and $\bar{v}$ at the surface are equal to their values at BOT mb, and that $(\partial \bar{T} / \partial t)_{\mathrm{L} \text { s. }}$, and $(\partial \bar{q} / \partial t)_{\mathbf{L} S}$ are equal to zero at the surface. $\bar{\omega}$ is defined by the surface pressure tendency $\partial p_{s} / \partial t$; we have neglected $v_{s} \cdot \nabla p_{s}$.

To obtain values for model levels above TOP mb by interpolation, the simplest approach is to assume that $\bar{T}, \bar{q}, \bar{u}$, and $\bar{v}$ at $20 \mathrm{~km}$ are equal to their values at TOP $\mathrm{mb}$, and that $\bar{\omega},(\partial \bar{T} / \partial t)_{\mathrm{L} . \mathrm{S}}$, and $(\partial \bar{q} / \partial t)_{\mathrm{L} \mathrm{S}}$ are equal to zero at $20 \mathrm{~km}$.

Radiative transfor calculations may require temperature, water vapor mixing ratio, and ozone mixing ratio profiles above $20 \mathrm{~km}$. The "standard mid-latitude summer atmosphere" profiles are available for this purpose, and can be found on the SCM Intercomparison web page under SCM Supporting Data Sets.

\subsubsection{Upper and lower boundary conditions}

The lower boundary is a land surface. For this intercomparison, values of surface sensible and latent heat flux are given as surface forcing for the models. The surface forcing terms are obtained by two methods: (1) site-wide averages of the observed fluxes by the ARM Energy Balance Bowen Ratio (EBBR) stations, and (2) site-wide integrated estimates of fluxes from the SiB2 land-surface model using ARM surface and satellite data streams as input and exercised on a $6.25 \mathrm{~km}$ grid (see Chris Doran's ARM project).

For diagnostic purposes, other surface quantities are provided hourly, including surface pressure, ground surface temperature, soil temperature and moisture, near-surface winds, downwelling solar and IR radiation, and precipitation. 
Radiative transfer calculations require the solar constant and the solar zenith angle (or its cosine). Due to the variation of the earth-sun distance $d$ from its mean value $d_{m}$, the solar "constant" $S$ is increased from its mean value of $S_{m}=1365 \mathrm{~W} / \mathrm{m}^{2}$. For J11ly 17 , the ratio $\left(d_{m} / d\right)^{2}=0.96814$, so $S=S_{m}\left(d_{m} / d\right)^{2}=1321.5 \mathrm{~W} / \mathrm{m}^{2}$. For August 5 , the ratio $\left(d_{m} / d\right)^{2}=0.97199$, so $S=S_{m}\left(d_{m} / d\right)^{2}=1326.8 \mathrm{~W} / \mathrm{m}^{2}$. The average of these two values of $S$ is $1324.1 \mathrm{~W} / \mathrm{m}^{2}$.

You may use the Fortran subroutine listed below (available via the SCM Intercomparison web page, under SCM Supporting Data Sets, and also via anonymous ftp in file solar.f) to calculate the cosine of the solar zenith angle. The central facility of the SGP CART is located at latitude $36^{\circ} 36^{\prime} \mathrm{N}$, longitude $97^{\circ} 29^{\prime} \mathrm{W}$, at an elevation of $315 \mathrm{~m}$ above sea level.

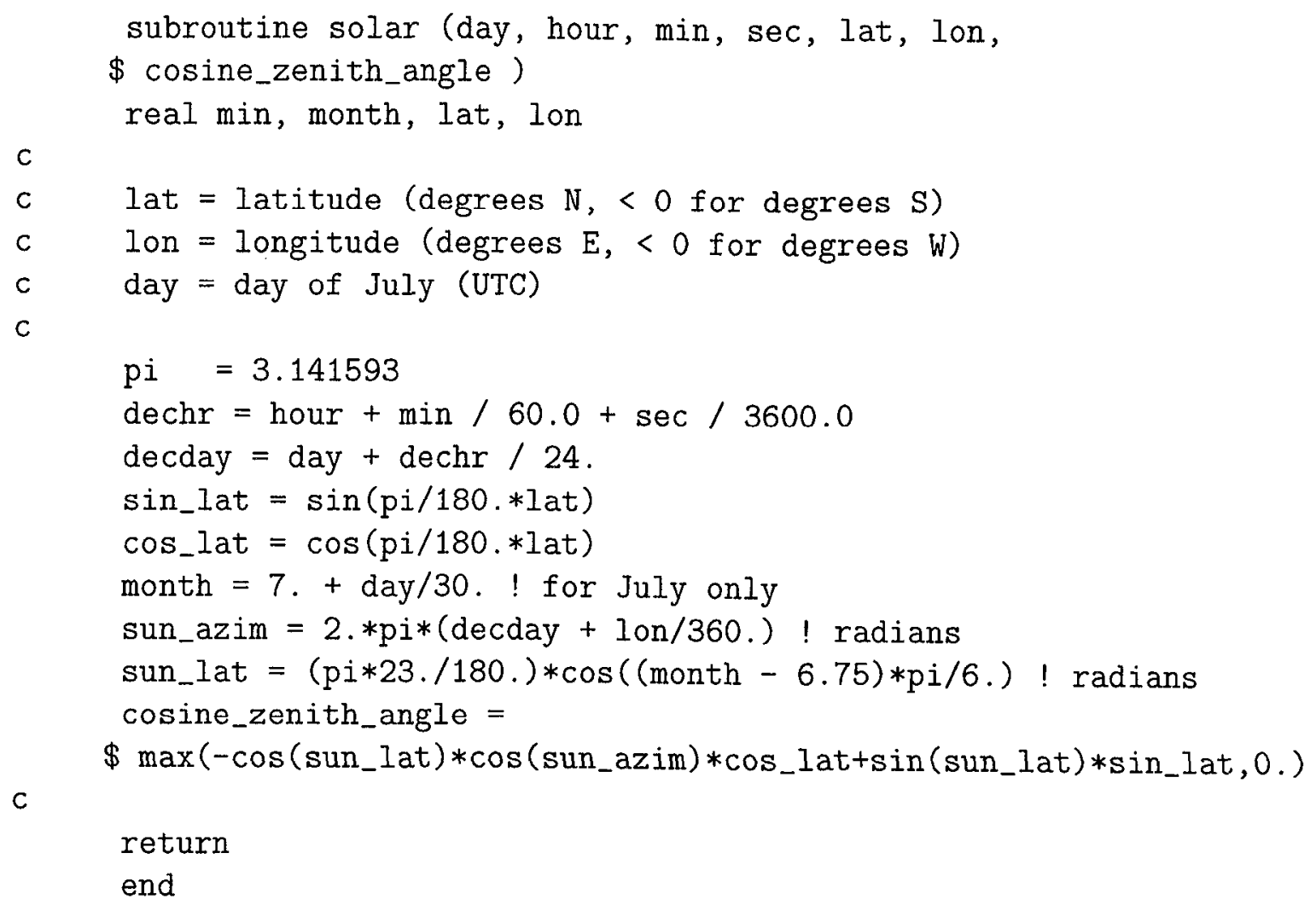

\subsubsection{Large-scale forcing}

The large-scale forcing terms in the SCM and CRM governing equations include the large-scale horizontal pressure gradient force, and the large-scale advective tendencies of horizontal momentum, potential temperature, water vapor mixing ratio, and hydrometeor mixing ratios. The CRM domains and SCM horizontal grid sizes are considered to be small compared to large-scale disturbances. In this case, the large-scale forcing will be approximately uniform over the model domains. For this reason, the large-scale forcing terms are defined to be functions of height and time only. The available large-scale forcing 
terms are based on observations averaged over the SGP CART region (about $365 \mathrm{~km}$ by $300 \mathrm{~km})$.

Temperature and water vapor mixing ratio The large-scale advective tendencies for temperature $T$ and water vapor mixing ratio $q$ are formally defined as

$$
\left(\frac{\partial \vec{T}}{\partial t}\right)_{\mathrm{L} . \mathrm{S}} \equiv-\overline{\mathbf{v}} \cdot \nabla \bar{T}-\bar{\omega} \frac{\partial \bar{T}}{\partial p}
$$

and

$$
\left(\frac{\partial \bar{q}}{\partial t}\right)_{\mathrm{L} \mathrm{S}} \equiv-\overline{\mathbf{v}} \cdot \nabla \bar{q}-\bar{\omega} \frac{\partial \bar{q}}{\partial p}
$$

where $\nabla$ is the horizontal del operator. Note that for potential temperature

$$
\left(\frac{\partial \bar{\theta}}{\partial t}\right)_{\mathrm{LS}}=\left(\frac{p_{0}}{\bar{p}}\right)^{R / c_{p}}\left(\frac{\partial \bar{T}}{\partial t}\right)_{\mathrm{L} \mathrm{S}} .
$$

SCMs and CRMs are unable to calculate $\nabla \bar{T}, \nabla \bar{q}$, or $\bar{\omega}$, so these must be specified from observations. In the models, $(\partial \bar{T} / \partial t)_{\mathrm{LS}}$ and $(\partial \bar{q} / \partial t)_{\mathrm{L} S}$ will be calculated using one of the following three methods.

Unfortunately, observations of the advective tendencies for hydrometeors are not available. This may have a significant impact on the simulation of middle and upper tropospheric stratiform clouds.

The lateral forcing will be specified in three different ways for the intercomparison runs: revealed forcing, vertically-forced, and relaxed to upstream forcing. Each is described below.

Specify the observed total advective tendency (revealed)

In this method,

$$
\left(\frac{\partial \bar{T}}{\partial t}\right)_{\mathrm{LS}}=\left(\frac{\partial \bar{T}}{\partial t}\right)_{\mathrm{LS} . \mathrm{T}}
$$

and

$$
\left(\frac{\partial \bar{q}}{\partial t}\right)_{\mathrm{LS}}=\left(\frac{\partial \bar{q}}{\partial t}\right)_{\mathrm{LST}}
$$

where $(\partial \bar{T} / \partial t)_{\mathrm{L} \mathrm{S} \mathrm{T}}$ and $(\partial \bar{q} / \partial t)_{\mathrm{L} \mathrm{S} \mathrm{T}}$ are the observed large-scale total advective tendencies for $T$ and $q$.

Specify the observed horizontal advective tendency (vertically-forced) In this method,

$$
\left(\frac{\partial \bar{T}}{\partial t}\right)_{\mathrm{LS}}=\left(\frac{\partial T}{\partial t}\right)_{\mathrm{L} \mathrm{SH}}-\bar{\omega} \frac{\partial \bar{T}}{\partial p}
$$


and

$$
\left(\frac{\partial \bar{q}}{\partial t}\right)_{\text {LS }}=\left(\frac{\partial \bar{q}}{\partial t}\right)_{\text {L S H. }}-\bar{\omega} \frac{\partial \bar{q}}{\partial p}
$$

where $(\partial \bar{T} / \partial t)_{\mathrm{LSH}}$ and $(\partial \bar{q} / \partial t)_{\mathrm{L} \text { S.H }}$ are the observed large-scale horizontal advective tendencies for $T$ and $q$, and $\bar{w}$ is the observed large-scale vertical velocity.

Relax toward the upstream value In this method,

$$
\left(\frac{\partial \bar{T}}{\partial t}\right)_{\text {L.S. }}=-\frac{\bar{T}-\bar{T}_{u}}{\tau_{a}}-\bar{\omega} \frac{\partial \bar{T}}{\partial p}
$$

and

$$
\left(\frac{\partial \bar{q}}{\partial t}\right)_{\mathrm{L} \mathrm{S.}}=-\frac{\bar{q}-\bar{q}_{u}}{\tau_{a}}-\bar{\omega} \frac{\partial \bar{q}}{\partial p}
$$

where $\bar{T}_{u}, \bar{q}_{u}$, and $\tau_{a}$ are the observed large-scale upstream temperature, mixing ratio, and advective time scale. Note, again, that upstream potential temperature can be obtained by

$$
\bar{\theta}_{u}=\left(\frac{p_{0}}{\bar{p}}\right)^{R / c_{p}} \bar{T}_{u}
$$

For this intercomparison, the advective time scale $\tau_{a}$ is defined as

$$
\tau_{a}=\frac{D}{2 \sqrt{u^{2}+v^{2}}}
$$

where $D$ is the distance across the SCM domain (specified here as $300 \mathrm{~km}$ ), and $u$ and $v$ are the wind components in the column from the analyses. The upstream values for temperature and water vapor mixing ratio are obtained from an average of grid point values from the NCEP Rapid Update Cycle (RUC) model that surround the SCM domain. An alternative method for estimating the upwind values is to equate the relaxation term to the horizontal advection term (i.e. for temperature), with the observed temperature used in place of the model-predicted value

$$
-\frac{\bar{T}_{o b s}-\bar{T}_{u}}{\tau_{a}}=\left(\frac{\partial \bar{T}}{\partial t}\right)_{\mathrm{L} \mathrm{S} \mathrm{H.}}
$$

After some manipulation, the upstream temperature is

$$
\bar{T}_{u}=\widetilde{T}_{o b s}+\tau_{a}\left(\frac{\partial \bar{T}}{\partial t}\right)_{\text {L.S H }}
$$

Similarly, the upstream water vapor mixing ratio is

$$
\bar{q}_{u}=\bar{q}_{o b s}+\tau_{a}\left(\frac{\partial \bar{q}}{\partial t}\right)_{\mathrm{LS} \mathrm{H}}
$$

Profile values are given at 10-mb intervals for LLNL objective analyses, and at 50-mb intervals for the SUNY variational analysis; both are given at 3-hour intervals. These values must be interpolated in pressure to the model's grid and in time to obtain values at each time step. 


\subsection{Multidimensional (CRM) Aspects}

\subsubsection{Domain size}

In order to simulate MCSs and to average out the "noise" produced by mesoscale convective systems in the large-scale statistics, a large horizontal domain is needed. A large domain is also necessary to be consistent with the size of the SGP CART. This is not a problem for 2D CRMs, but for 3D CRMs, the domain will be less extensive and the horizontal grid size will be larger.

\subsubsection{Vertical grid structure}

The following vertical grid structure is recommended for CRMs. SCMs may use other grids. The domain depth will be at least $20 \mathrm{~km}$. The vertical grid size nearest the surface will be $100 \mathrm{~m}$, stretching gradually to $500 \mathrm{~m}$ at $6 \mathrm{~km}$ above the surface, and remaining constant at $500 \mathrm{~m}$ from $6 \mathrm{~km}$ to $20 \mathrm{~km}$ above the surfacc. The grid lcvel hcights $z_{k}$ (in meters) between 0 and $6 \mathrm{~km}$ above the surface where the vertical velocity is defined are

$$
z_{k}=z_{s}+\left(c_{1}+c_{2} z_{k}^{\prime}\right) z_{k}^{\prime} \text { for } k=0,20
$$

where $z_{s}$ is the surface elevation above mean sea level, $z_{k}^{\prime}=k \Delta z^{\prime}, c_{1}=0.2982456142$, $c_{2}=1.169590643 \times 10^{-4}$, and $\Delta z^{\prime}=300 \mathrm{~m}$. The grid level heights between 0 and $6 \mathrm{~km}$ above the surface where the horizontal velocity and pressure are defined when using a staggered grid are

$$
z_{k}=z_{s}+\left(c_{1}+c_{2} z_{k}^{\prime}\right) z_{k}^{\prime} \text { for } k=1,20
$$

where $z_{k}^{\prime}=(k-0.5) \Delta z^{\prime}$. The grid level heights between 6 and $20 \mathrm{~km}$ above the surface where the vertical velocity is defined are

$$
z_{k}=z_{s}+(k-20) \Delta z+6000 \text { for } k=21,50
$$

where $\Delta z=500 \mathrm{~m}$. The grid level heights between 6 and $20 \mathrm{~km}$ above the surface where the horizontal velocity and pressure are defined are

$$
z_{k}=z_{s}+(k-20.5) \Delta z+6000 \text { for } k=21,50 .
$$

\subsubsection{Convection initiation}

The initial conditions will be horizontally uniform. Convection will be initiated by introducing small random perturbations in the temperature field ( $0.5 \mathrm{~K}$ maximum magnitude) in the subcloud layer for the first hour or so. Coarse-grid (i.e., 3D) models may need to introduce such perturbations during the entire simulation in order to trigger convection during undisturbed periods. 


\subsubsection{Boundary conditions}

The lateral boundary condition will be cyclic. This is consistent with imposing horizontallyuniform large-scale forcing. It is also convenient for budget studies.

Ideally, the upper boundary at $20 \mathrm{~km}$ should not reflect upward-propagating gravity wave energy. However, the particular approach used will be up to each modeler. Additional (sponge) layers may be added above $20 \mathrm{~km}$ (to perhaps $40 \mathrm{~km}$ ) to absorb gravity wave energy. 


\subsection{Numerical simulations}

Six simulations are described below. They cover an extended $2 \times 3$ matrix of two sources of forcing data (Barnes objective analysis and variational analysis), three specifications of the large-scale advective forcing, and two methods for estimating surface forcing. The basic $2 \times 3$ matrix uses the surface forcing obtained from averaged EBBR data. The extended runs (A2, D2, E2, and F2) use the surface forcing based on the SiB2 model values. For all CRM simulations, the $x$ coordinate is oriented east-west and increases toward the east. For all 2D CRM simulations, the domain is oriented east-west. For all CRM simulations, use a horizontal grid size of $2 \mathrm{~km}$. For all 2D CRM simulations, use a domain size of about $500 \mathrm{~km}$.

For each simulation, specify the large-scale advective tendencies for temperature and water vapor mixing ratio according to the appropriate description in section 2.1.3.

Use Forcing Data from Barnes Objective Analysis (LLNL)

Simulation A: Specify the observed total advective tendencies.

Simulation B: Specify the observed horizontal advective tendencies.

Simulation C: Relax toward the upstream values.

Simulation A2: Same as Simulation A, except with SiB2 surface forcing. Use Forcing Data from Variational Analysis (SUNY-SB, Zhang)

Simulation D: Specify the observed total advective tendencies.

Simulation E: Specify the observed horizontal advective tendencies.

Simulation F: Relax toward the upstream values.

Simulation D2: Same as Simulation D, except with SiB2 surface forcing.

Simulation E2: Same as Simulation E, except with SiB2 surface forcing.

Simulation F2: Same as Simulation F, except with SiB2 surface forcing.

\subsection{Obtaining Data and Submitting Results}

\subsubsection{Accessing and reading the files}

Data files for the SCM Intercomparison will be at the ARM Experiment Center. They are accessible by FTP, as follows:

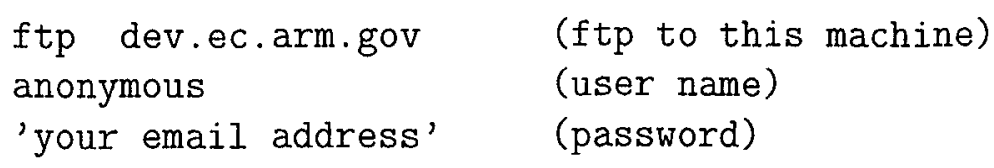


cd outgoing/scm_intercomp/case1_9507

*** in this base directory, you will find documentation and subdirectories with the desired netCDF and ASCII files ***

Vertical profiles of observed large-scalc temperature $\left(\bar{T}_{\text {obs }}\right)$, mixing ratio $\left(\bar{q}_{\text {obs }}\right)$, and velocity components $\left(\bar{u}_{\mathrm{obs}}, \bar{v}_{\mathrm{obs}}\right.$, and $\left.\bar{\omega}\right)$; the observed ground surface temperature $\left(\bar{T}_{s}\right)$ and the surface pressure $\left(\bar{p}_{0}\right)$; profiles of the observed large-scale total advective tendencies of temperature and mixing ratio $\left((\partial \bar{T} / \partial t)_{\mathrm{L} \mathrm{S.T}}\right.$ and $\left.(\partial \bar{q} / \partial t)_{\mathrm{LST}}\right)$, horizontal advective tendencies of temperature and mixing ratio $\left((\partial \bar{T} / \partial t)_{\mathrm{L} \mathrm{S.H}}\right.$ and $\left.(\partial \bar{q} / \partial t)_{\mathrm{L.S} \mathrm{H} .}\right)$, and upstream temperature $\left(\bar{T}_{u}\right)$, mixing ratio $\left(\bar{q}_{u}\right)$, and advective time scale $\left(\tau_{a}\right)$ are contained in files in subdirectory sgpforcing. 9507, with one file per day. Values are given for temperature, instead of potential temperature, for all terms except observed total advective tendency where values are given for both temperature (including effects of adiabatic compression) and potential temperature. Other temperature terms can be converted to potential terms using the conversion factor:

$$
\left(\frac{p_{0}}{\bar{p}}\right)^{R / c_{p}}
$$

where $p_{o}$ is $1000 \mathrm{mb}$. This conversion factor for each pressure level is included in the files.

Site-wide averages of surface values are included in ASCII files in subdirectory surface_data for the July 18 through August 3 period. The file names are:

$$
\begin{aligned}
& \text { sgpsfcflux_doran_csu.c1.950718.000000.asc } \\
& \text { sgpsf cmet_csu.c1.950718.000000.asc } \\
& \text { sgpsfcprcp_csu.c1.950718.000000.asc } \\
& \text { sgpsfcrad_ghan.c1.950718.000000.asc }
\end{aligned}
$$

Profilcs and arca-avcraged valucs from Zhang's variational analysis are contained in subdirectory varanal. Documentation and Fortran codes to read the data files are available there.

\subsubsection{Submitting output files}

Files containing results of your runs can be submitted via. FTP to the ARM Experiment Center. Modify the FTP procedures above by accessing a different directory to put your result files:

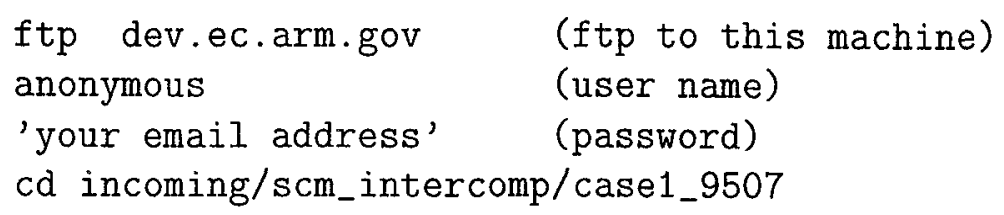

Once you are in that directory, you may create your own subdirectory using mkdir your_directory_name. For our convenience, use your last name for the directory name, unless you have another preference. Files are automatically removed from the FTP directory 
14 days after your submission, so we must transfer them to other long-term SCM directories within that time. It will help us if you send email to John Yio (johnyio@llnl.gov) and Ric Cederwall (rcederwall@llnl.gov) after you submit results so that we will make sure that your result files are handled in a timely manner. 


\section{Results to Submit}

The results to be submitted will consist of large-scale quantities unless otherwise noted. Large-scale is defined as both a space and time average. The space average is the horizontal average over the entire domain. The time average is the average over a 3-hour period, and should be based on "observations" taken frequently enough to avoid aliasing due to cloud-scale and mesoscale variability. Some quantities, such as surface rainfall, should be accumulated every time step. Unless noted otherwise, time averages should be obtained from observations taken no less often than every 15 or 20 minutes.

The large-scale quantities to be submitted will consist of time series and profiles at 3-hourly intervals. The time series will be largely based on quantities provided in more detail in the profiles, so the latter will be described first.

An overbar indicates a large-scale average. Unless explicitly defined as a large-scale quantity, a variable without a bar is a grid-scale average (i.e., a grid-point value). A grid-scale average may also be denoted by angle brackets. A grid-scale departure from the large-scale average is indicated by a single prime, so that $a^{\prime}=\bar{a}-a$. A subgrid-scale departure from the grid-scale average is indicated by a double prime.

The definitions of "cloud top height," "cloud top temperature," "clear" and "cloudy" columns, "rainy" columns, "echo top height" and "convective" and "stratiform" columns, follow the lists.

It is nearly certain that there will be revisions and additions to this list, both before and after the intercomparison workshop. To allow for such reprocessing, please save the following $2 \mathrm{D}$ or $3 \mathrm{D}$ CRM fields at all grid points every 15 or 20 minutes: temperature or potential temperature $(T$ or $\theta)$, all water substance mixing ratios $\left(q, q_{c}, q_{i}, q_{r}, q_{s}, q_{g}\right)$, the velocity components $(u, v, w)$ and the downwelling and upwelling infrared and solar radiative fluxes $\left(F_{L W}^{-}, F_{L W}^{+}, F_{S W}^{-}\right.$, and $\left.F_{S W}^{+}\right)$.

Instructions on how to submit the files containing the quantities described below have been outlined in Section 2.4.2 above. If you have questions, you may contact Ric Cederwall via e-mail at rcederwall@llnl.gov. We have chosen to use ASCII format for submitting results as a convenience for participants. We are considering use of netCDF format for future case studies, and will provide assistance and helpful utilities at that time. You may submit your results in netCDF format now if you prefer. Please put the profile data in a separate file from the time series (which themselves can go in three separate files). 


\subsection{Description of quantities}

Associated with each of the quantities below is a priority, in bold type and brackets $<>$. All quantities apply to SCMs and CRMs, unless denoted by a *, which means CRM only. The priorities are:

$\begin{array}{ll}1 & \text { required } \\ 2 & \text { recommended } \\ 3 & \text { optional }\end{array}$

\section{- Profiles}

Submit the results for each quantity listed below as a separate ASCII file in the special file format described in the section after the definitions. Include the time at the midpoint of the averaging interval $\bar{t}$ (hours, F6.1) and either (a) the pressure $p$ (mb, F7.3) or (b) the height $z$ (km, F7.3) as the $x$ and $y$ coordinates. Name each file as follows: simulation.pitem.modeler. For example, the file name "a.p2.krueger" indicates simulation $=a$, profile item $=2$ (temperature), and modeler $=$ krueger. To reduce the file sizes, suggested formats for each quantity are included in the descriptions below using Fortran notation.

1. $<\mathbf{1}>$ Height, $z(\mathrm{~km})$ (F7.3) if on constant pressure surface, or Pressure, $p(\mathrm{mb})$ (F7.3) if on constant height surface

2. $<\mathbf{1}>$ Temperature, $\bar{T}(\mathrm{~K})(\mathrm{F} 7.2)$

3. $<\mathbf{1}>$ Water vapor mixing ratio, $\bar{q}(\mathrm{~g} / \mathrm{kg})(\mathrm{F} 7.3)$

4. $<$ 2>Relative humidity, $R$ (unitless) (F6.3): $R=\bar{q} / q^{*}(\bar{T}, \bar{p})$, where $q^{*}(T, p)$ is the saturation mixing ratio over water.

5. $<\mathbf{1}>$ Cloud water (suspended liquid water) mixing ratio, $\overline{q_{c}}(\mathrm{~g} / \mathrm{kg})(\mathrm{F} 7.4)$

6. $<\mathbf{1}>$ Cloud ice (suspended ice) mixing ratio, $\overline{q_{i}}(\mathrm{~g} / \mathrm{kg})(\mathrm{F} 7.4)$

7. $<\mathbf{3}>$ Rain (falling liquid water) mixing ratio, $\overline{q_{r}}(\mathrm{~g} / \mathrm{kg})(\mathrm{F} 7.4)$

8. $<3>$ Snow (slow-falling ice) mixing ratio, $\overline{q_{s}}(\mathrm{~g} / \mathrm{kg})(\mathrm{F} 7.4)$

9. $<3>$ Graupel (fast-falling ice) mixing ratio, $\overline{q_{g}}(\mathrm{~g} / \mathrm{kg})(\mathrm{F} 7.4)$

10. $\left\langle\mathbf{1}^{*}>\right.$ Cloud fraction, $\bar{\sigma}$ (unitless) (F6.3): At each grid point, $\sigma=1$ if $q_{c}+q_{i}>$ $0.01 q^{*}(\bar{T}, \bar{p})$; otherwise, $\sigma=0$.

11. $<\mathbf{2}>$ Horizontal wind velocity in $\mathrm{x}$-direction, $\bar{u}(\mathrm{~m} / \mathrm{s})(\mathrm{F} 7.2)$

12. $<2>$ Horizontal wind velocity in y-direction, $\bar{v}(\mathrm{~m} / \mathrm{s})(\mathrm{F} 7.2)$

13. $<\mathbf{1}>$ Apparent heat source, $Q_{1 C}$ (K/day) (F7.2):

$$
Q_{1 C} \equiv Q_{1}-Q_{R}=\left(\frac{\bar{p}}{p_{0}}\right)^{R / c_{p}}\left[\frac{\partial \bar{T}}{\partial t}-\left(\frac{\partial \bar{T}}{\partial t}\right)_{\mathrm{I} S}\right]-Q_{R}
$$


where

$$
\left(\frac{\partial \bar{T}}{\partial t}\right)_{\text {L.S }} \equiv-\overline{\mathbf{v}} \cdot \nabla \bar{T}-\bar{\omega} \frac{\partial \bar{T}}{\partial p}
$$

is the large-scale advective tendency (or "forcing") which is specified from observations.

14. <1>Apparent moisture sink, $Q_{2}$ (K/day) (F7.2):

$$
Q_{2}=-\frac{L}{c_{p}}\left[\frac{\partial \bar{q}}{\partial t}-\left(\frac{\partial \bar{q}}{\partial t}\right)_{\mathrm{L} \mathrm{S.}}\right]
$$

where

$$
\left(\frac{\partial \bar{q}}{\partial t}\right)_{\mathrm{LS} .} \equiv-\overline{\mathbf{v}} \cdot \nabla \bar{q}-\bar{\omega} \frac{\partial \bar{q}}{\partial p}
$$

is the large-scale advective tendency (or "forcing") which is specified from observations.

15. $<3>$ Convective $Q_{1 C}, Q_{1 C}^{\mathrm{c}}$ (K/day) (F7.2): Contribution to $Q_{1 C}$ from the "convective" columns.

16. $<3>$ Stratiform $Q_{1 C}, Q_{1 C}^{\mathrm{s}}$ (K/day) (F7.2): Contribution to $Q_{1 C}$ from the "stratiform" columns.

17. $<3>$ Convective $Q_{2}, Q_{2}^{\mathrm{c}}$ (K/day) (F7.2): Contribution to $Q_{2}$ from the "convective" columns.

18. $<3>$ Stratiform $Q_{2}, Q_{2}^{\text {s }}$ (K/day) (F7.2): Contribution to $Q_{2}$ from the "stratiform" columns.

19. $<\mathbf{1}>$ Radiative heating rate, $Q_{R}$ (K/day) (F7.2)

20. $<1>$ Solar (short-wave) radiative heating rate, $Q_{R}^{\mathrm{SW}}(\mathrm{K} /$ day) $(\mathrm{F} 7.2)$

21. $<1>$ Infrared (long-wave) radiative heating rate, $Q_{R}^{\mathrm{LW}}$ (K/day) (F7.2)

22. $<1>$ Clear radiative heating rate, $Q_{R}^{\mathrm{clr}}$ (K/day) (F7.2): The average radiative heating rate in the "clear" columns.

23. $<1>$ Cloudy radiative heating rate, $Q_{R}^{\text {cld }}(\mathrm{K} /$ day $)(\mathrm{F} 7.2)$ : The average radiative heating rate in the "cloudy" columns.

24. $<2>$ Cloud mass flux, $M_{c}\left(\mathrm{mb} \mathrm{s}^{-1}\right)(\mathrm{F} 8.5): M_{c}=M_{u}-M_{d}$

25. $<2>$ Updraft cloud mass flux, $M_{u}\left(\mathrm{mb} \mathrm{s}^{-1}\right)(\mathrm{F} 7.5)$ :

$$
M_{u}=\frac{\sum_{j} \sigma \omega^{+}}{\sum_{j}}
$$

where $j$ is the grid point column index, $\sigma$ is the cloud fraction (defined previously), $\omega^{+}=\omega$ if $\omega<0$, otherwise, $\omega^{+}=0$. 
26. $<2>$ Downdraft cloud mass flux, $M_{d}\left(\mathrm{mb} \mathrm{s}^{-1}\right)(\mathrm{F} 7.5): M_{d}=M_{d s}+M_{d u}$, where $M_{d s}$ and $M_{d u}$ are the saturated and unsaturated downdraft cloud mass fluxes:

$$
M_{d s}=\frac{\sum_{j} \sigma \omega^{-}}{\sum_{j}}
$$

and

$$
M_{d u}=\frac{\sum_{j} \sigma_{p} \omega^{-}}{\sum_{j}} .
$$

Here, $\omega^{-}=|\omega|$ if $\omega>0$, otherwise, $\omega^{-}=0$. Also, $\sigma_{p}=1$ if $\sigma=0$ and $q_{r}+q_{s}+q_{g}>P$, with $P=0.1 \mathrm{~g} / \mathrm{kg}$; otherwise, $\sigma_{p}=0$.

27. $<3>$ Fractional area of updraft cores, $\overline{\sigma_{u}}$ (unitless) (F6.3): A "core" exists if $|\omega|>W$, with $W=0.1 \mathrm{mb} / \mathrm{s}$. Thus,

$$
\overline{\sigma_{u}}=\frac{\sum_{j} \sigma_{u}}{\sum_{j}}
$$

where $\sigma_{u}=1$ if $\omega<-W$; otherwise, $\sigma_{u}=0$.

28. $<\mathbf{3}>$ Fractional area of downdraft cores, $\overline{\sigma_{d}}$ (unitless) (F6.3):

$$
\overline{\sigma_{d}}=\frac{\sum_{j} \sigma_{d}}{\sum_{j}}
$$

where $\sigma_{d}=1$ if $\omega>W$; otherwise, $\sigma_{d}=0$.

29. $<3>$ Average core updraft speed, $\omega_{u}(\mathrm{mb} / \mathrm{s})($ F7.3):

$$
\omega_{u}=\frac{\sum_{j} \sigma_{u} \omega}{\sum_{j} \sigma_{u}}
$$

30. $<3>$ Average core downdraft speed, $\omega_{d}(\mathrm{mb} / \mathrm{s})(\mathrm{F} 7.3)$ :

$$
\omega_{d}=\frac{\sum_{j} \sigma_{d} \omega}{\sum_{j} \sigma_{d}}
$$

\section{- Time series}

Submit the results for each of the three groups of quantities listed below as a separate ASCII file. Name each file as follows: simulation.tgroup.modeler. For example, the file name "a.t1.krueger" indicates simulation=a, time series group=1, and modeler $=$ krueger.

In the first line of each file, please include a comment identifier (\#k), the filename, and, optionally, any further distinguishing characteristics. Follow this with the results; use one line for each time. This is an example of the file format:

\#k a.t1.krueger 2D baseline run 
... (results for all group 1 quantities at the first time) ...

$\cdot$

... (results for all group 1 quantities at the last time) ...

Suggested formats for each quantity are included in the descriptions below using Fortran notation. So that each line includes no more than 80 characters (necessary for e-mail transmission), simply write each line using the suggested formats. For example, the format for each line for group 1 is (F6.1,2F7.2,F6.2,3F7.2,2F6.1, 2F8.4). For group 2, it is (F6.1,2F7.1,2F6.1,F7.1,2F6.1,2F6.3,F6.2,2E10.3), and for group 3 it is (F6.1,3E10.3,3F7.2,3F6.3).

If the files are transmitted via $\mathrm{ftp}$, all that is required is that the results for each time are contained within one record (a line ending with a carriage return) of any length, and that each quantity (including the first) be preceded by at least one space. The format can be fixed point or E- (exponential) format.

\section{Group 1:}

1. $<\mathbf{1}>$ Time of mid-point of averaging interval, $\bar{t}(\mathrm{~h})(\mathrm{F} 6.1)$

2. $<\mathbf{1}>$ Surface skin temperature, SST (K) (F7.2)

3. $<3>$ Near-surface dry static energy, $\overline{s_{0}}(\mathrm{~kJ} / \mathrm{kg})(\mathrm{F} 7.2): s=c_{p} T+g z$. "Nearsurface" is the first model level above the surface.

4. $<3>$ Near-surface water vapor mixing ratio, $\overline{q_{0}}(\mathrm{~g} / \mathrm{kg})(\mathrm{F} 6.2)$

5. $<3>$ Near-surface moist static energy, $\overline{h_{0}}(\mathrm{~kJ} / \mathrm{kg})(\mathrm{F} 7.2): h=s+L q$.

6. $<3>$ Near-surface horizontal wind velocity in x-direction, $\overline{u_{0}}(\mathrm{~m} / \mathrm{s})(\mathrm{F} 7.2)$

7. $<3>$ Near-surface horizontal wind velocity in y-direction, $\overline{v_{0}}(\mathrm{~m} / \mathrm{s})(\mathrm{F} 7.2)$

8. $<\mathbf{1}>$ Surface turbulent flux of sensible heat, $\overline{\left(F_{s}\right)_{0}}\left(\mathrm{~W} / \mathrm{m}^{2}\right)(\mathrm{F} 6.1): F_{s} \equiv$ $\bar{\rho} c_{p}\left(\frac{\bar{p}}{p_{0}}\right)^{R / c_{p}}\left\langle w^{\prime \prime} T^{\prime \prime}\right\rangle$.

9. $<1>$ Surface turbulent flux of latent heat, $\overline{L\left(F_{q}\right)_{0}}\left(\mathrm{~W} / \mathrm{m}^{2}\right)(\mathrm{F} 6.1): F_{q} \equiv$ $\bar{\rho}\left\langle w^{\prime \prime} q^{\prime \prime}\right\rangle$.

10. $<\mathbf{2}>$ Surface turbulent flux of horizontal momentum component in $\mathrm{x}$-direction, $\overline{\left(F_{u}\right)_{0}}\left(\mathrm{nt} / \mathrm{m}^{2}\right)(\mathrm{F} 8.4): F_{u} \equiv \bar{\rho}\left\langle u^{\prime \prime} w^{\prime \prime}\right\rangle$.

11. $\leq 2>$ Surface turbulent flux of horizontal momentum component in y-direction, $\overline{\left(F_{v}\right)_{0}}\left(\mathrm{nt} / \mathrm{m}^{2}\right)(\mathrm{F} 8.4): F_{v} \equiv \bar{\rho}\left\langle v^{\prime \prime} w^{\prime \prime}\right\rangle$.

\section{Group 2:}

1. $<\mathbf{1}>$ Time of mid-point of averaging interval, $\bar{t}(\mathrm{~h})(\mathrm{F} 6.1)$

2. $<1>$ Surface downwelling solar radiative flux, $\overline{\left(F_{S W}\right)_{0}}\left(\mathrm{~W} / \mathrm{m}^{2}\right)(\mathrm{F} 7.1)$ 
3. $<\mathbf{1}>$ Surface upwelling solar radiative flux, $\overline{\left(F_{S W}^{+}\right)_{0}}\left(\mathrm{~W} / \mathrm{m}^{2}\right)(\mathrm{F} 7.1)$

4. $<\mathbf{1}>$ Surface downwelling infrared radiative flux, $\overline{\left(F_{L W}^{-}\right)_{0}}\left(\mathrm{~W} / \mathrm{m}^{2}\right)(\mathrm{F} 6.1)$

5. $<1>$ Surface upwelling infrared radiative flux, $\overline{\left(F_{L W}^{+}\right)_{0}}\left(\mathrm{~W} / \mathrm{m}^{2}\right)(\mathrm{F} 6.1)$

6. $<\mathbf{1}>$ TOA (top of atmosphere) downwelling solar radiative flux, $\overline{\left(F_{S W}^{-}\right)_{T}}\left(\mathrm{~W} / \mathrm{m}^{2}\right)$ (F7.1)

7. $<\mathbf{1}>$ TOA upwelling solar radiative flux, $\overline{\left(F_{S W}^{+}\right)_{T}}\left(\mathrm{~W} / \mathrm{m}^{2}\right)(\mathrm{F} 6.1)$

8. $<1>$ TOA upwelling infrared radiative flux (OLR), $\overline{\left(F_{L W}^{+}\right)_{T}}\left(\mathrm{~W} / \mathrm{m}^{2}\right)(\mathrm{F} 6.1)$

9. $<1>$ Cloud amount, $\overline{A_{\text {cld }}}$ (unitless) (F6.3): Fraction of columns which are "cloudy" for CRMs. For SCMs, this depends on cloud layer overlap assumptions.

10. $<\mathbf{2}>$ Cold cloud top area, $\overline{A_{\text {cld }}^{\text {cold }}}$ (unitless) (F6.3): Fraction of columns for which the "cloud top temperature" is less than $245 \mathrm{~K}$.

11. $<1>$ Precipitable water, $\overline{\mathrm{PW}}\left(\mathrm{kg} / \mathrm{m}^{2}\right)(\mathrm{F} 6.2): \mathrm{PW}=\int_{0}^{z_{T}} \bar{\rho} q d z$, where $z_{T}$ is the model top height.

12. $<\mathbf{1}>$ Cloud liquid water path, $\overline{\mathrm{LWP}}\left(\mathrm{kg} / \mathrm{m}^{2}\right)(\mathrm{E} 10.3)$ : LWP $=\int_{0}^{z_{T}} \bar{\rho} q_{c} d z$.

13. $<1>$ Cloud ice path, $\overline{\mathrm{IWP}}\left(\mathrm{kg} / \mathrm{m}^{2}\right)(\mathrm{E} 10.3): \mathrm{IWP}=\int_{0}^{z_{T}} \bar{\rho} q_{i} d z$.

\section{Group 3:}

1. $<\mathbf{1}>$ Time of mid-point of averaging interval, $\bar{t}(\mathrm{~h})(\mathrm{F} 6.1)$

2. $<3>$ Vcrtically integrated rain, $\overline{\mathrm{RP}}\left(\mathrm{kg} / \mathrm{m}^{2}\right)(\mathrm{E} 10.3): \mathrm{RP}=\int_{0}^{z_{T}} \bar{\rho} q_{r} d z$.

3. $<3>$ Vertically integrated snow, $\overline{\mathrm{SP}}\left(\mathrm{kg} / \mathrm{m}^{2}\right)(\mathrm{E} 10.3): \mathrm{SP}=\int_{0}^{z_{T}} \bar{\rho} q_{s} d z$.

4. $<3>$ Vertically integrated graupel, $\overline{\mathrm{GP}}\left(\mathrm{kg} / \mathrm{m}^{2}\right)(\mathrm{E} 10.3): \mathrm{GP}=\int_{0}^{z_{T}} \bar{\rho} q_{g} d z$.

5. $<1>$ Surface rainfall rate, $\bar{P}(\mathrm{~mm} /$ day) (F7.2): Calculate from the surface rain accumulated over every time step.

6. $<\mathbf{2}>$ Convective surface rainfall rate, $\overline{P_{c}}(\mathrm{~mm} /$ day) $(\mathrm{F} 7.2)$ : The contribution to $\bar{P}$ from the "convective" columns.

7. $<2>$ Stratiform surface rainfall rate, $\overline{P_{s}}$ (mm/day) $(\mathrm{F} 7.2)$ : The contribution to $\bar{P}$ from the "stratiform" columns.

8. $<3>$ Rain fractional area, $\overline{A_{r}}$ (unitless) (F6.3): Fraction of columns which are "rainy."

9. $<\mathbf{1}>$ Convective fractional arca, $\overline{A_{c}}$ (unitless) (F6.3): Fraction of columns which are "convective."

10. $<\mathbf{1}>$ Stratiform fractional area, $\overline{A_{s}}$ (unitless) (F6.3): Fraction of columns which are "stratiform." 


\subsection{Definitions (for CRMs only)}

- The "cloud top height," $z_{c}$, is the height where the top-downward integrated cloud water path equals $C=0.02 \mathrm{~kg} / \mathrm{m}^{2}$. I.e., $z_{c}$ satisfies

$$
\begin{gathered}
\int_{z_{c}}^{z_{T}} \bar{\rho}\left(q_{c}+q_{i}\right) d z=C . \\
\text { If } \int_{0}^{z_{T}} \bar{\rho}\left(q_{c}+q_{i}\right) d z<C \text { then } z_{c}=0 .
\end{gathered}
$$

- The "cloud top temperature," $T_{B}$, is the temperature at "cloud top height."

- In a "cloudy" column, the "cloud top height" is greater than the surface height.

- If a column is not "cloudy", it is "clear."

- In a "rainy" column, the rain mixing ratio $\left(q_{r}\right)$ is greater than $E=10^{-3} \mathrm{~g} / \mathrm{kg}$ at the first model level above the surface. This is approximately equivalent to defining a "rainy" column as one in which the radar reflectivity factor $Z$ exceeds 0.1 (dBZ exceeds -10) or the rainfall rate exceeds $0.01 \mathrm{~mm} / \mathrm{hr}$.

- The "echo top height, " $z_{e}$ is the maximum height where $q_{r}+q_{s}+q_{g}>E=10^{-3}$ $\mathrm{g} / \mathrm{kg}$.

- "Convective" column: To allow comparison to TOGA COARE radar data analyzed by Tom Rickenbach, this definition relies on the surface rainfall rate $P$ only. It is based on the method of Steiner et al. (1995: J. Appl. Meteor., 34, 1978-2007). Other more physically-based definitions have been proposed. Because the radar data has 2-km horizontal resolution, $P$ must first be mapped onto a 2-km grid. In the following, "grid point" refers to a 2-km-wide grid point. The criteria:

- Intensity: Any grid point with $P \geq 10 \mathrm{~mm} / \mathrm{hr}$ is classified as "convective."

- Peakedness: Any grid point with $P$ that exceeds the "background" rate $P_{b}$ by at least a factor of two is classified as "convective." The "background" rate is the average of the rates in the "rainy" $(P>0.01 \mathrm{~mm} / \mathrm{hr})$ grid points within an 11-km radius (the 10 adjacent 2-km-wide grid points for 2D models).

- Surrounding area: For each grid point identified as a convective center by at least one of the above criteria, all surrounding grid points within an intensitydependent "convective radius" about the grid point are included as convective. For $P_{b}<1.2 \mathrm{~mm} / \mathrm{hr}$, the radius is $1 \mathrm{~km}$ (no additional grid points are classified as convective). For $1.2 \mathrm{~mm} / \mathrm{hr} \leq P_{b}<2.5 \mathrm{~mm} / \mathrm{hr}$, the radius is $2 \mathrm{~km}$ (for a $2 \mathrm{D}$ model, 1 additional 2-km-wide grid point is classified as convective). For 2.5 $\mathrm{mm} / \mathrm{hr} \leq P_{b}<5 \mathrm{~mm} / \mathrm{hr}$, the radius is $3 \mathrm{~km}$ (for a $2 \mathrm{D}$ model, 2 additional grid points are classified as convective). For $5 \mathrm{~mm} / \mathrm{hr} \leq P_{b}<10 \mathrm{~mm} / \mathrm{hr}$, the radius is $4 \mathrm{~km}$ (for a 2D model, 3 additional grid points are classified as convective). For $P_{b} \geq 10 \mathrm{~mm} / \mathrm{hr}$, the radius is $5 \mathrm{~km}$ (for a $2 \mathrm{D}$ model, 4 additional grid points are classified as convective). 
- "Stratiform" column: All "rainy" $(P>0.01 \mathrm{~mm} / \mathrm{hr})$ 2-km grid points not classified as convective are "stratiform" grid points.

\subsection{Special file format}

This ASCII text file format represents a 2D array $f(x, y)$ with $x$ and $y$ coordinates. Such files should be in the format shown below.

\# filename simulation_description variable_description comments ny $\mathrm{nx}$

$f_{-} \max f_{-}$min

$\mathrm{y}(1) \mathrm{y}(2) \quad \ldots \quad \mathrm{y}(\mathrm{ny})$

$\mathrm{x}(1) \mathrm{x}(2) \quad \cdots \quad \mathrm{x}(\mathrm{nx})$

$f(x(1), y(1)) \quad f(x(2), y(1)) \ldots . f(x(n x), y(1)) f(x(1), y(2)) \ldots$

$f(x(1), y(n x)) \quad f(x(2), y(n x)) \ldots . f(x(n x), y(n x))$

The file may consist of lines (records) of any convenient length. The first line is a comment. Values for the numbers may be integers, floating point, or exponential format, separated by spaces, tabs, or end-of-lines. Maximum and minimum values define the range of $f$. Simply enter 0 for these; they will be reset after all the files are received. 


\section{Model Description}

In order to compare results from diffcrent models, workshop participants arc asked to complete the following description relevant for simulations performed for the intercomparison. 'T'his description will help to build up the workshop report at the final stage.

- Scientist name

Affiliation

Address

Email address

Fax Number

- Model type (1D, 2D, 3D)

- Numerical Domain

Domain size in $\mathrm{x}$-direction:

Domain size in $\mathrm{y}$-direction:

Domain size in z-direction:

Number of grid points in $\mathrm{x}$-direction:

Number of grid points in y-direction:

Number of grid points in z-direction:

Grid size in $\mathrm{x}$-direction:

Grid size in y-direction:

Grid size in z-direction (if stretched please specify):

- Time step

- Numerical Technique

Numerical method (finite-difference, spectral, etc.):

Advection scheme and its order of accuracy:

Time scheme and its order of accuracy:

Dynamical equations (elastic, anelastic, etc.):

Numerical diffusion (type, order, magnitude of coefficient)

Upper boundary condition (Sponge layer, specification, ...):

Other information 
- Physical Parameterizations:

Surface flux parameterization for heat, moisture, momentum:

Longwave radiation parameterization:

Shortwave radiation parameterization:

How were radiative fluxes above the computational domain handled?

Microphysical (2D/3D models) or cloud/convective (1D model) parameterization: type, number of hydrometeor classes, ...

Turbulence closure scheme (turbulence closure type, variables predicted and diagnosed by the turbulence closure, closure for turbulent length scale, ...)

Other information

- Documentation: Please provide references that more fully describe your model, if available. 


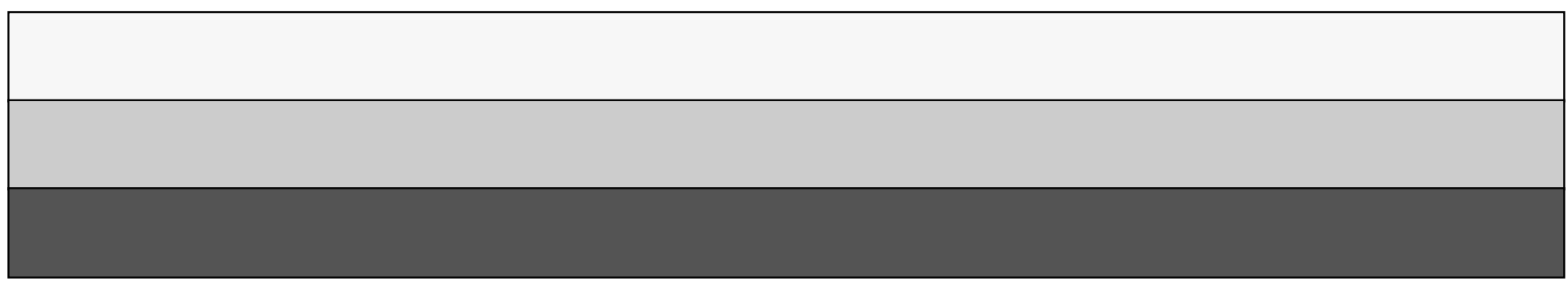

